\title{
Non-transverse factorizing fields and entanglement in finite spin systems
}

\author{
M. Cerezo, R. Rossignoli, N. Canosa \\ Departamento de Física-IFLP, Universidad Nacional de La Plata, C.C.67, La Plata (1900), Argentina
}

\begin{abstract}
We determine the conditions for the existence of non-transverse factorizing magnetic fields in general spin arrays with anisotropic $X Y Z$ couplings of arbitrary range. It is first shown that a uniform maximally aligned completely separable eigenstate can exist just for fields $\boldsymbol{h}_{s}$ parallel to a principal plane and forming four straight lines in field space, with the alignment direction different from that of $\boldsymbol{h}_{s}$ and determined by the anisotropy. Such state always becomes a non-degenerate ground state (GS) for sufficiently strong (yet finite) fields along these lines, in both ferromagnetic (FM) and antiferromagnetic (AFM) type systems. In AFM chains, this field coexists with the nontransverse factorizing field $\boldsymbol{h}_{s}^{\prime}$ associated with a degenerate Néel-type separable GS, which is shown to arise at a level crossing in a finite chain. It is also demonstrated for arbitrary spin that pairwise entanglement reaches full range in the vicinity of both $\boldsymbol{h}_{s}$ and $\boldsymbol{h}_{s}^{\prime}$, vanishing at $\boldsymbol{h}_{s}$ but approaching small yet finite side-limits at $\boldsymbol{h}_{s}^{\prime}$, which are analytically determined. The behavior of the block entropy and entanglement spectrum in their vicinity is also analyzed.
\end{abstract}

PACS numbers: 75.10.Jm, 03.67.Mn, 03.65.Ud, 64.70.Tg

\section{INTRODUCTION}

The ground state (GS) of strongly interacting spin systems immersed in a magnetic field $\boldsymbol{h}$ can exhibit, under certain conditions, the remarkable phenomenon of factorization [1], i.e., of becoming a product of single spin states. Such exact factorization can occur at finite fields despite the strong couplings existing between the spins, albeit at very specific values (and orientation) of the field. In the seminal work of ref. [1, it was shown that antiferromagnetic (AFM) chains with first neighbor $X Y Z$ couplings possess a separable Néel-type GS (NGS) if the field vector lies on the surface of an ellipsoid determined by the couplings. Factorization was then investigated in other models with transverse fields 22 20], with a general formalism for describing factorization introduced and discussed in 79 .

In 10, 11, 14] we have shown that in finite $X Y Z$ chains, the transverse factorizing field (TFF) $\boldsymbol{h}_{z s}$ pointing along a principal axis $(z)$ corresponds actually to the last GS $S_{z}$ parity transition (level crossing). The ensuing separable GS is two-fold degenerate, breaking a basic symmetry of the Hamiltonian $\left(S_{z}\right.$ parity). The non-transverse factorizing fields (NTFF) $\boldsymbol{h}_{s}^{\prime}$ of [1] will be shown to also belong to this class in finite cyclic chains, i.e., they arise at the last GS level crossing and determine a degenerate separable GS breaking translational invariance (TI). In finite systems the underlying mechanism of factorization in these cases is the existence of separable linear combinations of the symmetry preserving entangled crossing states.

In this work we first determine the general conditions for exact factorization under non-transverse fields. It is then shown that a uniform non-degenerate separable GS (UGS) does exist at a field $\boldsymbol{h}_{s}$ which does not belong in general to the ellipsoid of ref. [1], and does not correspond to a level crossing. This GS actually arises in both AFM and FM-type systems, even for couplings of arbitrary range provided there is a fixed anisotropy ratio, but only for fields parallel to a principal plane, with the set of fields $\boldsymbol{h}_{s}$ forming four straight lines. Factorization emerges here from the splitting of the degenerate separable eigenstates existing at the TFF $\boldsymbol{h}_{z s}$. Unlike $\boldsymbol{h}_{s}^{\prime}, \boldsymbol{h}_{s}$ can be arbitrarily strong, allowing the separation of the UGS from the remaining spectrum. This enables an easy preparation of an exactly separable state, which can be useful for quantum information applications (a product initial state is assumed in the standard model of quantum computation 21]).

A second but not less important aspect of factorization is that it corresponds to an entanglement transition: In the transverse case, the factorizing field is, remarkably, the point where pairwise entanglement reaches full range in its immediate vicinity, and changes its type 5, 6, 10, 11, 14. We had previously shown that the entanglement between any two spins reaches in a finite chain weak yet finite common side-limits at the transverse field $\boldsymbol{h}_{z s}$, irrespective of separation or coupling range 10, 11, , arising from the entangled crossing states. This type of limit also occurs at the NTFF $\boldsymbol{h}_{s}^{\prime}$ of ref. [1], as will be shown. But in addition, we will prove that pairwise entanglement also reaches full range at the vicinity of the NTFF $\boldsymbol{h}_{s}$ leading to a non-degenerate UGS. Here the entanglement between any two spins, though 0 at $\boldsymbol{h}_{s}$, is turned on as $\boldsymbol{h}_{s}$ is approached from either side, with the concurrence vanishing then linearly with $\left|\boldsymbol{h}-\boldsymbol{h}_{s}\right|$. The underlying reason is essentially the monogamy of entanglement 22, 23, which prevents distant pairs from becoming entangled if first or close neighbors are strongly entangled. In the vicinity of $\boldsymbol{h}_{s}$, close neighbor entanglement decreases strongly, allowing the emergence of weak yet non-zero entanglement between distant pairs. The behavior of the block entanglement entropy in the vicinity of the NTFF will be also analyzed. It will be shown to vanish essentially quadratically at $\boldsymbol{h}_{s}$, while at $\boldsymbol{h}_{s}^{\prime}$ it will approach finite side-limits in a finite chain, which will be analytically determined. The entanglement spectrum will indicate, as expected, just one nonzero eigenvalue at 
$\boldsymbol{h}_{s}$, although at the side-limits of $\boldsymbol{h}_{s}^{\prime}$ two nonzero eigenvalues will remain.

The general equations for non-transverse factorizing fields and its uniform and Néel-type solutions are derived and discussed in section [II, whereas entanglement together with illustrative results for the pairwise concurrence, block entropy, entanglement spectrum and magnetization in FM and AFM chains with $X Y$ and $X Y Z$ couplings under non-transverse fields are discussed in section III. Conclusions are derived in IV.

\section{FACTORIZATION IN NON-TRANSVERSE FIELDS}

\section{A. General Equations}

We consider an array of $n$ spins $S_{i}$ not necessarily equal, interacting through $X Y Z$ Heisenberg couplings of arbitrary range in the presence of a general magnetic field $\boldsymbol{h}^{i}=\left(h_{x}^{i}, h_{y}^{i}, h_{z}^{i}\right)$, not necessarily uniform. The Hamiltonian reads

$$
H=-\sum_{i, \mu} h_{\mu}^{i} S_{i}^{\mu}-\frac{1}{2} \sum_{i \neq j, \mu} J_{\mu}^{i j} S_{i}^{\mu} S_{j}^{\mu},
$$

where $i, j$ label the sites in the array, $S_{i}^{\mu}, \mu=x, y, z$, the spin components at site $i$ and $J_{\mu}^{i j}$ the coupling strengths between spins $i$ and $j\left(J_{\mu}^{i j} \geq 0\right.$ corresponds to the FM case whilst $J_{\mu}^{i j} \leq 0$ to the AFM case). In the transverse case $h_{x}^{i}=h_{y}^{i}=0 \forall i, H$ conserves the $S_{z}$-parity $P_{z}=$ $\exp \left[\imath \pi \sum_{i}\left(S_{i}^{z}+S_{i}\right)\right]\left(\left[H, P_{z}\right]=0\right)$. This symmetry no longer holds for non-transverse fields.

We now determine the general conditions for which $H$ possesses a completely separable eigenstate of the form

$$
|\Theta\rangle=\otimes_{i=1}^{n} R_{i}\left|0_{i}\right\rangle, \quad R_{i}=\exp \left[-\imath \phi_{i} S_{i}^{z}\right] \exp \left[-\imath \theta_{i} S_{i}^{y}\right],
$$

where $\left|0_{i}\right\rangle$ denotes the local state with maximum spin along $z\left(S_{i}^{z}\left|0_{i}\right\rangle=S_{i}\left|0_{i}\right\rangle\right)$ and $R_{i}$ rotates this state to direction $\boldsymbol{n}_{i}=\left(\sin \theta_{i} \cos \phi_{i}, \sin \theta_{i} \sin \phi_{i}, \cos \theta_{i}\right)$. The equation $H|\Theta\rangle=E_{\Theta}|\Theta\rangle$ leads, after writing $H$ in terms of the rotated spins $S_{i}^{\mu^{\prime}}=R_{i} S_{i}^{\mu} R_{i}^{\dagger}$, to:

I) The field independent equations

$$
\begin{aligned}
& J_{y}^{i j}\left(\cos \phi_{i} \cos \phi_{j}-\cos \theta_{i} \sin \phi_{i} \cos \theta_{j} \sin \phi_{j}\right)= \\
& J_{x}^{i j}\left(\cos \theta_{i} \cos \phi_{i} \cos \theta_{j} \cos \phi_{j}-\sin \phi_{i} \sin \phi_{j}\right) \\
& +J_{z}^{i j} \sin \theta_{i} \sin \theta_{j}, \\
& J_{y}^{i j}\left(\cos \theta_{i} \sin \phi_{i} \cos \phi_{j}+\cos \phi_{i} \cos \theta_{j} \sin \phi_{j}\right)= \\
& J_{x}^{i j}\left(\cos \theta_{i} \cos \phi_{i} \sin \phi_{j}+\sin \phi_{i} \cos \theta_{j} \cos \phi_{j}\right),
\end{aligned}
$$

which are also independent of spin and are responsible for cancelling all elements connecting $|\Theta\rangle$ with two-spin excitations, and
II) The field dependent equations

$$
\begin{aligned}
& h_{z}^{i} \sin \theta_{i}-\cos \theta_{i}\left(h_{x}^{i} \cos \phi_{i}+h_{y}^{i} \sin \phi_{i}\right)= \\
& \sum_{j \neq i} S_{j}\left[\cos \theta_{i} \sin \theta_{j}\left(J_{x}^{i j} \cos \phi_{i} \cos \phi_{j}+J_{y}^{i j} \sin \phi_{i} \sin \phi_{j}\right)\right. \\
& \left.-J_{z}^{i j} \sin \theta_{i} \cos \theta_{j}\right], \\
& h_{x}^{i} \sin \phi_{i}-h_{y}^{i} \cos \phi_{i}= \\
& \sum_{j \neq i} S_{j} \sin \theta_{j}\left[-J_{x}^{i j} \sin \phi_{i} \cos \phi_{j}+J_{y}^{i j} \cos \phi_{i} \sin \phi_{j}\right]
\end{aligned}
$$

which cancel all elements connecting $|\Theta\rangle$ with single spin excitations and are just the mean field stationary equations $\partial_{\theta_{i}}\langle H\rangle=0, \partial_{\phi_{i}}\langle H\rangle=0$, where

$$
\langle H\rangle \equiv\langle\Theta|H| \Theta\rangle=-\sum_{i}\left\langle\boldsymbol{S}_{i}\right\rangle \cdot\left(\boldsymbol{h}^{i}+\frac{1}{2} \sum_{j} \boldsymbol{J}^{i j}\left\langle\boldsymbol{S}_{j}\right\rangle\right),
$$

with $\left\langle\boldsymbol{S}_{i}\right\rangle=S_{i} \boldsymbol{n}_{i}$ and $\boldsymbol{J}^{i j}$ a diagonal matrix of elements $J_{\mu}^{i j}$. If Eqs. (3)-(4) are satisfied $\forall i, j$, Eqs. (5) - (6) determine the set of factorizing fields.

In terms of the alignment directions $\boldsymbol{n}_{i} \equiv \boldsymbol{n}_{i}^{z^{\prime}}$ and the orthogonal unit vectors $\boldsymbol{n}_{i}^{y^{\prime}}=\left(-\sin \phi_{i}, \cos \phi_{i}, 0\right), \boldsymbol{n}_{i}^{x^{\prime}}=$ $\left(\cos \theta_{i} \cos \phi_{i}, \cos \theta_{i} \sin \phi_{i},-\sin \theta_{i}\right)$, we may also express Eqs. (3)-(4) as

$$
\begin{aligned}
& \boldsymbol{n}_{i}^{x^{\prime}} \cdot \boldsymbol{J}^{i j} \boldsymbol{n}_{j}^{x^{\prime}}=\boldsymbol{n}_{i}^{y^{\prime}} \cdot \boldsymbol{J}^{i j} \boldsymbol{n}_{j}^{y^{\prime}}, \\
& \boldsymbol{n}_{i}^{x^{\prime}} \cdot \boldsymbol{J}^{i j} \boldsymbol{n}_{j}^{y^{\prime}}=-\boldsymbol{n}_{i}^{y^{\prime}} \cdot \boldsymbol{J}^{i j} \boldsymbol{n}_{j}^{x^{\prime}},
\end{aligned}
$$

which imply $J_{x^{\prime} x^{\prime}}^{i j}=J_{y^{\prime} y^{\prime}}^{i j}$ and $J_{x^{\prime} y^{\prime}}^{i j}=-J_{y^{\prime} x^{\prime}}^{i j}$ when writing the coupling in (10) in terms of the rotated spins $S_{i}^{\mu^{\prime}}$, i.e., $\sum_{\mu} J_{\mu}^{i j} S_{i}^{\mu} S_{j}^{\mu}=\sum_{\mu, \nu} J_{\mu^{\prime} \nu^{\prime}}^{i j} S_{i}^{\mu^{\prime}} S_{j}^{\nu^{\prime}}$. And Eqs. (5) - (6) become

$$
\boldsymbol{n}_{i}^{\mu^{\prime}} \cdot\left(\boldsymbol{h}^{i}+\sum_{j} \boldsymbol{J}^{i j}\left\langle\boldsymbol{S}_{j}\right\rangle\right)=0, \mu^{\prime}=x^{\prime}, y^{\prime},
$$

implying that $\boldsymbol{h}^{i}$ should cancel the components of $\sum_{j} \boldsymbol{J}^{i j}\left\langle\boldsymbol{S}_{j}\right\rangle$ orthogonal to the alignment direction, such that

$$
\boldsymbol{h}^{i}+\sum_{j} \boldsymbol{J}^{i j}\left\langle\boldsymbol{S}_{j}\right\rangle \propto \boldsymbol{n}_{i} .
$$

The general solution for the NTFF at site $i$ is then

$$
\boldsymbol{h}_{s}^{i}=\boldsymbol{h}_{\|}^{i}+\boldsymbol{h}_{\perp}^{i},
$$

where $\boldsymbol{h}_{\|}^{i}=h_{\|}^{i} \boldsymbol{n}_{i}$ is an arbitrary field parallel to the local alignment direction, which just shifts the energy (7), and

$$
\boldsymbol{h}_{\perp}^{i}=-\sum_{j}\left[\boldsymbol{J}^{i j}\left\langle\boldsymbol{S}_{j}\right\rangle-\boldsymbol{n}_{i}\left(\boldsymbol{n}_{i} \cdot \boldsymbol{J}^{i j}\left\langle\boldsymbol{S}_{j}\right\rangle\right)\right],
$$

is a field orthogonal to the alignment direction $\left(\boldsymbol{n}_{i}\right.$. $\boldsymbol{h}_{\perp}^{i}=0$ ), representing the NTFF of lowest magnitude. Nonetheless, a finite $h_{\|}^{i}$ will be normally required in order that $|\Theta\rangle$ be a GS (see sec. II.C). Let us remark, finally, that Eqs. (8)-(12) remain valid for general couplings $\sum_{\mu, \nu} J_{\mu \nu}^{i j} S_{i}^{\mu} S_{j}^{\nu}$ in (1). 


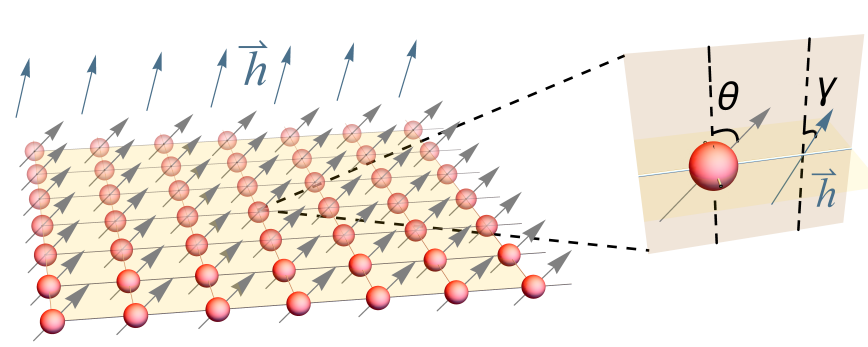

FIG. 1. (Color online) Schematic plot of the uniform solution.

\section{B. Uniform solution}

Eqs. (3)-(6) (or (8)-(10) ) are quite general and describe a wide range of interesting scenarios. We examine first the possibility of a uniform solution with $\theta_{i}=\theta$, $\phi_{i}=\phi \forall i$ (Fig. 10), such that $|\Theta\rangle$ is a maximum spin state: $\left|\left\langle\sum_{i} \boldsymbol{S}_{i}\right\rangle\right|=\sum_{i} S_{i}$. Such solution preserves TI and then has the possibility to be a non-degenerate GS in systems with this invariance under a uniform field.

Eq. (4) becomes $\left(J_{x}^{i j}-J_{y}^{i j}\right) \cos \theta \sin 2 \phi=0$, implying, if $J_{x}^{i j}-J_{y}^{i j} \neq 0$ for at least one pair, that the spin vector $\left\langle\boldsymbol{S}_{i}\right\rangle$ should be parallel to a principal plane $(x z$ if $\phi=0$, $y z$ if $\phi=\pi / 2$ and $x y$ if $\theta=\pi / 2$ ). Without loss of generality, we can assume $\phi=0$ (the other choices are rotations of this case). Eq. (3) then leads to

$$
\cos ^{2} \theta=\frac{J_{y}^{i j}-J_{z}^{i j}}{J_{x}^{i j}-J_{z}^{i j}}=\chi,
$$

if $J_{x}^{i j} \neq J_{z}^{i j}$, implying a constant anisotropy ratio $\chi$ for these pairs, and an isotropic coupling $J_{\mu}^{i j}=J^{i j} \forall \mu$ if $J_{x}^{i j}=J_{z}^{i j}$. The condition $0 \leq \chi \leq 1$ imposes the restriction

$$
J_{x}^{i j} \geq J_{y}^{i j} \geq J_{z}^{i j} \text { or } J_{x}^{i j} \leq J_{y}^{i j} \leq J_{z}^{i j} .
$$

Eqs. (13)- (14) entail that the $J_{\mu}^{i j}$ should be of the form

$$
J_{\mu}^{i j}=J^{i j}+r^{i j} J_{\mu}
$$

with the $J_{\mu}$ 's satisfying (14). The state $|\Theta\rangle$ will then depend just on $\chi=\frac{J_{y}-J_{z}}{J_{x}-J_{z}}$, being independent of the coupling range determined by $J^{i j}$ and $r^{i j}$. Notice that Eq. (13) leads to four possible alignment directions in the $x z$ plane, corresponding to the solutions $\pm \theta$ and $\pm(\pi-\theta)$, with $\theta \in(0, \pi / 2)$.

We remark that in the fully isotropic case $r_{i j}=0 \forall i, j$ in (15) (rotationally invariant coupling), $\theta$ and $\phi$ remain obviously arbitrary under Eqs. (3)-(4), whereas in the $X X$ case $J_{x}^{i j}=J_{y}^{i j} \forall i j$ (coupling invariant under any rotation around the $z$ axis), Eq. (4) is trivially satisfied while (3) leads to $\sin \theta=0$ if $J_{x}^{i j} \neq J_{z}^{i j}$ for at least one pair, in agreement with (13), implying alignment just in the $z$ direction. We will focus in what follows on the anisotropic case $0<\chi<1$, where the alignment direction is non-trivial $(\theta \in(0, \pi / 2))$.
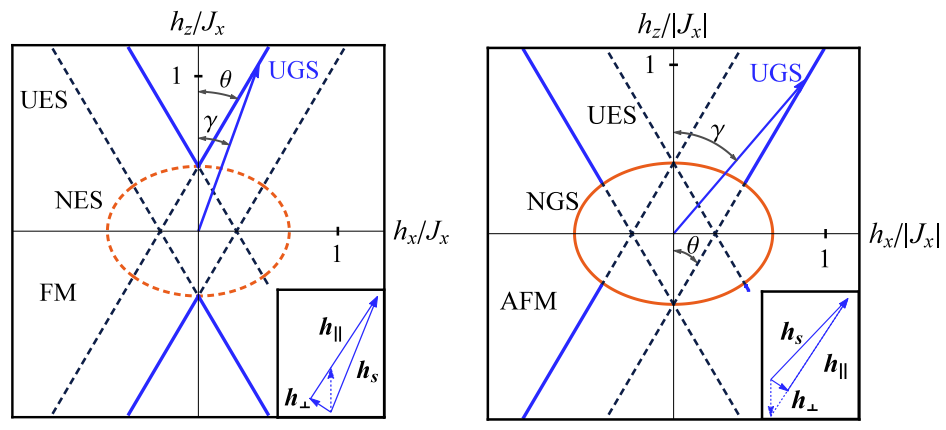

FIG. 2. (Color online) Factorizing fields for ferromagnetic (left) and antiferromagnetic (right) $X Y Z$ chains in the $x z$ principal plane of the field space. Solid straight lines depict the fields determining a uniform ground state (UGS), whereas the dashed straight lines those determining uniform excited eigenstates (UES). The ellipse depicts the fields corresponding to the Néel-type ground state (NGS, solid lines) or excited eigenstate (NES, dashed line). The plot corresponds to $J_{z}=0$ and $J_{x}>0(<0)$ in the FM (AFM) case, with $0<J_{y} / J_{x}<1$. The arrow indicates a direction of the external field along which one (FM) or two (AFM) GS factorizing fields are encountered as its magnitude increases. The field direction $\boldsymbol{n}_{\gamma}$ differs from the spin alignment direction $\boldsymbol{n}_{\theta}$. The insets depict the decomposition (18) of the non-transverse factorizing field for the UGS in both diagrams, with the dashed arrow indicating the transverse factorizing field $\boldsymbol{h}_{z s}$.

For $\phi=0$, Eqs. (6) (or (10) imply $h_{y}^{i}=0$, i.e., the field at each site should be parallel to the corresponding principal plane $(x z)$. Eq. (5) then becomes

$$
h_{z}^{i} \sin \theta-h_{x}^{i} \cos \theta=h_{\perp}^{i}
$$

where

$$
h_{\perp}^{i}=\sin \theta \cos \theta \sum_{j \neq i} S_{j}\left(J_{x}^{i j}-J_{z}^{i j}\right) .
$$

Setting $\boldsymbol{n}_{\theta}=\boldsymbol{n}_{i}=(\sin \theta, 0, \cos \theta)$ and $\boldsymbol{n}_{\theta}^{\perp}=$ $(-\cos \theta, 0, \sin \theta)$, Eqs. (16)- (17) imply that the NTFF is given by

$$
\boldsymbol{h}_{s}^{i}=h_{\|}^{i} \boldsymbol{n}_{\theta}+h_{\perp}^{i} \boldsymbol{n}_{\theta}^{\perp}
$$

in agreement with (11), with $h_{\|}^{i}$ arbitrary and $h_{\perp}^{i} \boldsymbol{n}_{\theta}^{\perp}$ orthogonal to the alignment direction. Eqs. (17)-18) give rise to a family of NTFF lying along four straight lines (Fig. 2), one for each alignment direction.

Note that field and spin directions cannot be parallel if $h_{\perp}^{i} \neq 0$ : At fixed field direction $\boldsymbol{n}_{\gamma}=(\sin \gamma, 0, \cos \gamma)$, i.e., $\boldsymbol{h}_{s}^{i}=h_{s}^{i}(\gamma) \boldsymbol{n}_{\gamma}$, Eqs. (18) leads to

$$
h_{s}^{i}(\gamma)=\frac{h_{\perp}^{i}}{\sin (\theta-\gamma)},
$$

which diverges for $\gamma \rightarrow \theta$. When the four values of $\theta$ are considered, Eq. (19) leads to two distinct values of $\left|\boldsymbol{h}_{s}^{i}\right|$ at fixed $\gamma \neq \pm \theta$, which merge at the principal axes (Fig. 2). 
For $\gamma=0$, we recover from (19) the TFF 10, 11

$$
h_{z s}^{i}=h_{s}^{i}(0)=\frac{h_{\perp}^{i}}{\sin \theta}
$$

which is the solution of (16) for $h_{x}^{i}=0$. We can then also express Eq. (18) as $\left(\boldsymbol{n}_{z}=(0,0,1)\right)$

$$
\boldsymbol{h}_{s}^{i}=h^{i} \boldsymbol{n}_{\theta}+h_{z s}^{i} \boldsymbol{n}_{z},
$$

where $h^{i}=h_{\|}^{i}-h_{\perp}^{i} / \tan \theta$. Hence, we can also consider $\boldsymbol{h}_{s}^{i}$ as the sum of the TFF $\boldsymbol{h}_{z s}^{i}=h_{z s}^{i} \boldsymbol{n}_{z}$ plus a non-transverse field of arbitrary magnitude $h_{i}$ along the spin alignment direction $\boldsymbol{n}_{\theta}$, which just shifts the energy $E_{\Theta}$.

In systems with TI (i.e., infinite or cyclic), $S_{i}=S$ and $h_{\perp}^{i}=h_{\perp} \forall i$, implying a uniform factorizing field $h_{s}(\gamma)$ at fixed orientation $\gamma$. Nonetheless, Eqs. (21)- (19) show that the uniform solution remains feasible even in the absence of TI, provided the $h_{\mu}^{i}$ at each site can be controlled independently. In particular, in open finite uniform chains or lattices with short range couplings, the uniform separable solution requires just border corrections to the otherwise uniform bulk factorizing field.

\section{Uniform ground state}

For the uniform solution, the energy (7) becomes

$$
\begin{aligned}
E_{\Theta} & =-\frac{1}{2} \sum_{i, j} S_{i} S_{j}\left(J_{x}^{i j}-J_{y}^{i j}+J_{z}^{i j}\right)-\sum_{i} S_{i} h_{\|}^{i} \\
& =-\frac{1}{2} \sum_{i, j} S_{i} S_{j}\left(J_{x}^{i j}+J_{y}^{i j}-J_{z}^{i j}\right)-\sum_{i} S_{i} h^{i} .
\end{aligned}
$$

It is then apparent that $|\Theta\rangle$ will be $G S$ if the fields $h_{\|}^{i} \boldsymbol{n}_{\theta}$ (or equivalently $h^{i} \boldsymbol{n}_{\theta}$ ) along the spin alignment direction are sufficiently strong, since no other state has an energy which decreases more rapidly with the applied field. Therefore, a transition to this uniform separable GS (UGS) will always arise as $h_{\|}^{i}$ increases, in both FM or AFM-type systems, as can be appreciated in Fig. 2 (transition from dashed to solid along the straight lines). Before this transition, $|\Theta\rangle$ is an excited eigenstate (no other state can increase its energy more rapidly for decreasing $h^{i}$ s).

We now show that if Eq. (13) is satisfied and, $\forall i, j$,

$$
J_{x}^{i j} \geq\left|J_{y}^{i j}\right|,
$$

such transition occurs at the TFF $\boldsymbol{h}_{z s}^{i}$, i.e., $|\Theta\rangle$ will be GS $\forall h_{i} \geq 0$ in (21) (left panel in Fig. 2).

Proof: We first note that if $\phi_{i}=0$ and $\theta_{i}=\theta \forall i$, Eq. (2) leads to

$|\Theta\rangle=\otimes_{i}\left(\sum_{k=0}^{2 S_{i}}\left(\begin{array}{c}2 S_{i} \\ k\end{array}\right)^{1 / 2} \cos ^{2 S_{i}-k} \frac{\theta}{2} \sin ^{k} \frac{\theta}{2}\left|k_{i}\right\rangle\right)$, where $S_{i}^{z}\left|k_{i}\right\rangle=\left(S_{i}-k\right)\left|k_{i}\right\rangle$. Eq. (24) implies that the interaction in $H$ will contain just negative or zero off-diagonal elements in the standard basis $\left\{\otimes_{i}\left|k_{i}\right\rangle\right\}$, as seen by writing (10) in terms of $S_{i}^{ \pm}=S_{i}^{x} \pm i S_{i}^{y}$. The same holds for
$H$ if $h_{y}^{i}=0$ and $h_{x}^{i} \geq 0 \forall i$. A GS with expansion coefficients real and of the same sign in this basis will then exist, as different signs will not decrease $\langle H\rangle$. But such GS cannot be orthogonal to $|\Theta\rangle$ if $\theta \in(0, \pi)$ (implying $h^{i} \geq 0$ in (21) if $h_{x}^{i} \geq 0$ ), so that it must coincide with $|\Theta\rangle$ when $|\Theta\rangle$ is an exact eigenstate. The case $h_{x}^{i} \leq 0$ can be reduced to the previous one by a rotation of angle $\pi$ around the $z$ axis, which leaves the rest of $H$ unchanged.

Besides, in the transverse case $h_{i}=0 \forall i$, the states $|\Theta\rangle$ and $|-\Theta\rangle=P_{z}|\Theta\rangle$, obtained for $\theta= \pm|\theta|$, become degenerate (Eq. (23)). The TFF $\boldsymbol{h}_{z s}^{i}$ determines then a pair of degenerate UGS $| \pm \Theta\rangle$ when (24) holds [10], and the addition of a field parallel to $\boldsymbol{n}_{\theta}\left(\boldsymbol{n}_{-\theta}\right)$ removes this degeneracy, leaving just $|\Theta\rangle(|-\Theta\rangle)$ as GS. The transition to the UGS takes then place at $\boldsymbol{h}_{z s}^{i}$.

The gap to the first excited state can then be made arbitrarily large by increasing the fields $h_{i}$ (Eq. (23)). Note that the similar case $J_{z}^{i j} \geq\left|J_{y}^{i j}\right| \forall i, j$ can be reduced to the previous one after a $\pi / 2$ rotation around the $y$ axis. Hence, in this case the transition takes place at the transverse field along $x, h_{x s}^{i}=h_{\perp}^{i} / \cos \theta=h_{z s}^{i} \tan \theta$.

\section{Néel-type solutions.}

In addition to the uniform solution, other solutions of Eqs. (3)- (6) can exist, which break TI. This is the case of the Néel-type separable eigenstates determined in 1] for the AFM chain with first neighbor couplings in a uniform field $\left(J_{i j}=0, r^{i j}=\delta_{i, j \pm 1}\right.$ in (14), with $J_{\mu} \leq 0$ for $\mu=x, y, z)$, where $\theta_{i}, \phi_{i}$ have alternating values. In a finite cyclic chain (with an even number $n$ of spins), such solution must then be two-fold degenerate, arising at the crossing of two non-separable TI eigenstates. The mechanism is then similar to that of the TFF for the uniform solution 10]. The associated NTFF $\boldsymbol{h}_{s}^{\prime}$ points to the surface of an ellipsoid [1], given for $S=1 / 2$ by

$$
\begin{aligned}
\frac{h_{s x}^{\prime 2}}{\left(J_{x}+J_{y}\right)\left(J_{x}+J_{z}\right)}+\frac{h_{s y}^{\prime 2}}{\left(J_{y}+J_{z}\right)\left(J_{y}+J_{x}\right)}+ & \frac{h_{s z}^{\prime 2}}{\left(J_{z}+J_{x}\right)\left(J_{z}+J_{y}\right)}=1 .
\end{aligned}
$$

Within the $x z$ plane, $\boldsymbol{h}_{s}^{\prime}=h_{s}^{\prime}(\gamma) \boldsymbol{n}_{\gamma}$ describes an ellipse (Fig. 2), satisfying

$$
\left|h_{s}^{\prime}(\gamma)\right|^{2}=\frac{\left(J_{x}+J_{z}\right)\left(J_{x}+J_{y}\right)\left(J_{z}+J_{y}\right)}{\left(J_{x}+J_{y}\right) \cos ^{2} \gamma+\left(J_{z}+J_{y}\right) \sin ^{2} \gamma} .
$$

While in a FM-type chain such solution also exists but corresponds to an excited eigenstate (left panel in Fig. 2), in the AFM case it is a GS which coexists with the previous UGS in the $x z$ field plane (right panel). For instance, they can arise for the same field orientation at different field magnitudes. This possibility is related with the existence of different solutions for the local unitary operations which can leave an eigenstate invariant in the treatment of 79 . Moreover, the point where the 

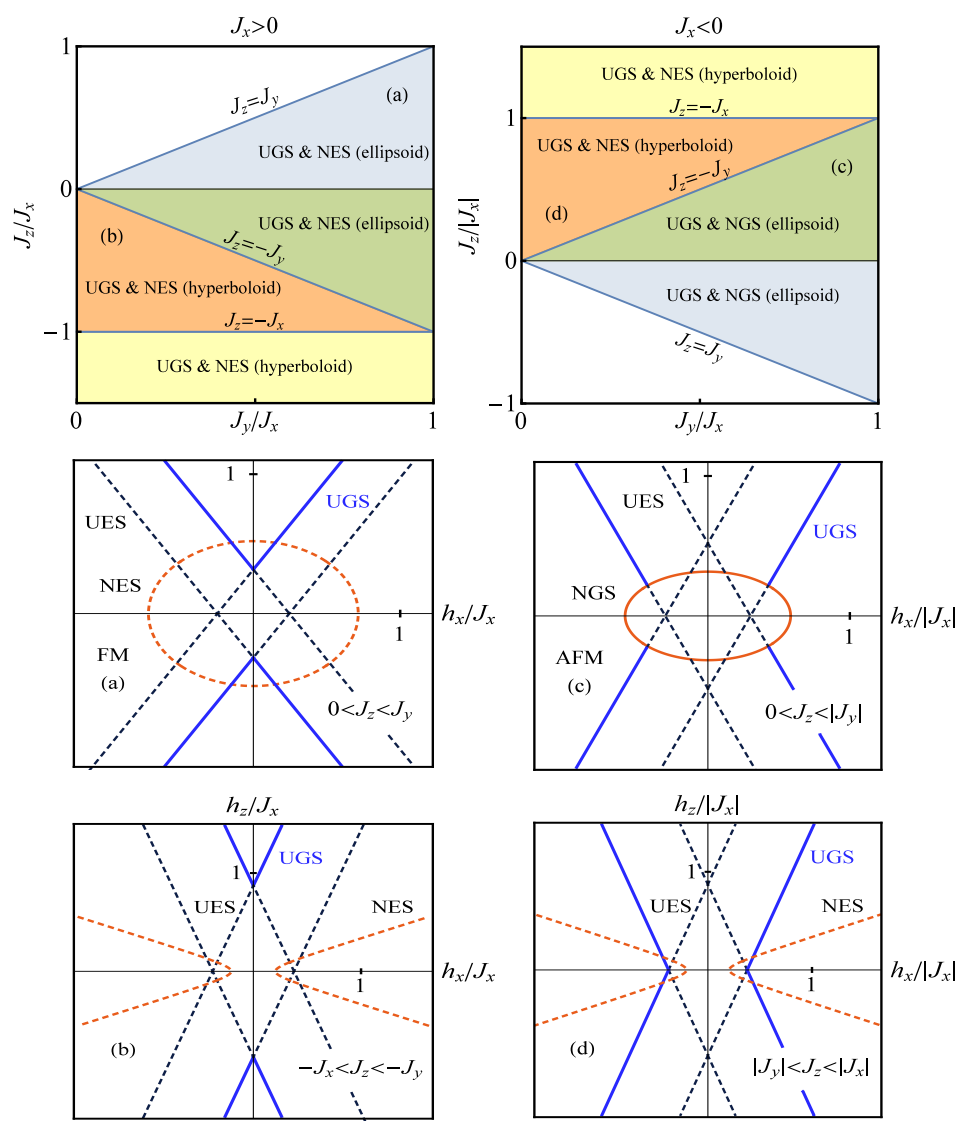

FIG. 3. (Color online) Factorization diagram (top panels) and factorizing fields (central and bottom panels) for $X Y Z$ couplings satisfying Eq. (14), with $J_{x}>0(<0)$ in the left (right) panels and $J_{y}$ of the same sign as $J_{x}$. The different combinations of couplings are indicated (see also text). For $J_{x}<J_{y}<0$, the Néel separable eigenstate ceases to be GS when $J_{z} \geq-J_{y}$, although the uniform separable eigenstate remains GS for appropriate fields, as seen in the top and bottom right panels. Eq. 25) may determine an hyperboloid when the couplings have different signs, as seen in the top and bottom panels.

straight line of the uniform solution crosses the ellipsoid $\left(\boldsymbol{h}_{s}=\boldsymbol{h}_{s}^{\prime}\right)$ is precisely that beyond which the uniform solution becomes GS (at this point the Néel-type solution becomes uniform, coinciding with the UGS). Hence, within the first quadrant, the UGS arises for field angles $0 \leq \gamma<\theta$ in the FM case but $\theta<\gamma \leq \gamma_{\mathrm{m}}$ in the AFM case, with

$$
\tan \gamma_{m}=\frac{J_{x}+J_{y}}{J_{y}+J_{z}} \tan \theta .
$$

Within this window, the GS of the AFM chain exhibits then two distinct factorizing fields as $\boldsymbol{h}$ increases at fixed $\boldsymbol{n}_{\gamma}$ (right panel in Fig. 22), a result which has not yet been reported.

Eq. (25) may also determine an hyperboloid when $J_{x}$, $J_{y}$ and $J_{z}$ do not have all the same signs, as shown in Fig. 3. where all (non-equivalent) possible combinations of couplings for the case (14) are considered. When $\left|J_{z}\right|$ increases from 0 , the diagrams of Fig. 2 2 remain essentially unchanged if $\left|J_{z}\right|<\left|J_{y}\right|$ (central panels in Fig. 3), both in the proper FM and AFM cases (all couplings of the same sign) as well as in those where $J_{z}$ has the opposite sign of $J_{x}$. However, when $\left|J_{z}\right|>\left|J_{y}\right|$ (with (14) still holding, e.g. $J_{x}<J_{y}<0$ and $J_{z}>-J_{y}$ ), the ellipsoid turns into an hyperboloid and the Néel-type state ceases to be $G S$ in the originally AFM case ( $J_{x}$ and $J_{y}$ negative), as indicated in the top and bottom right panels. Yet the uniform separable eigenstate remains GS for both $J_{x}>0$ and $J_{x}<0$ (blue lines in the bottom panels). This is still the case when $\left|J_{z}\right|$ increases beyond $\left|J_{x}\right|$, as indicated in the top panels. We just mention that the cases $J_{x}>$ $0>J_{y}>J_{z}$ and $J_{x}<0<J_{y}<J_{z}$ are equivalent, respectively, to $J_{z}>0>J_{y}>J_{x}$ and $J_{x}>J_{y}>0>$ $J_{z}$, after rotation around the $y$ axis. Furthermore, cases where Eq. (14) does not hold can be transformed to the present situation by a suitable rotation.

\section{ENTANGLEMENT IN THE VICINITY OF FACTORIZATION}

\section{A. Entanglement in the vicinity of the UGS}

Let us now discuss entanglement in the vicinity of the NTFF $\boldsymbol{h}_{s}$ leading to the uniform GS $|\Theta\rangle$. For simplicity we consider here a uniform field $\boldsymbol{h}$ in a spin $S$ system with TI, where the reduced two-spin density matrix $\rho_{i j}=\operatorname{Tr}_{\overline{i, j}}|G S\rangle\langle G S|\left(\operatorname{Tr}_{\overline{i, j}}\right.$ denotes the trace over the complementary subsystem) depends just on the separation between the two spins. This reduced state will be in general a mixed state when $|G S\rangle$ is entangled. And such mixed state is said to be entangled if it cannot be written as a convex mixture of product states $\rho_{i} \otimes \rho_{j}$ 24, i.e., if it cannot be generated by local operations and classical communication 21].

We first show that pairwise entanglement reaches full range in the vicinity of the factorizing field $\boldsymbol{h}_{s}$.

Proof: For $\boldsymbol{h}$ close to $\boldsymbol{h}_{s}$, the GS can be obtained by considering first order perturbative corrections to $|\Theta\rangle$ :

$$
\begin{aligned}
|G S\rangle & \approx|\Theta\rangle+\sum_{\nu} \frac{\left\langle\nu\left|\left(\boldsymbol{h}-\boldsymbol{h}_{s}\right) \cdot\left(\sum_{i} \boldsymbol{S}_{i}\right)\right| \Theta\right\rangle}{E_{\nu}-E_{\Theta}}|\nu\rangle \\
& =|\Theta\rangle+\left(\alpha \sum_{i}{S_{i}^{-{ }^{\prime}}}^{\prime}+\sum_{i, j} \beta_{i j}{S_{i}^{-{ }^{\prime}}}^{{ }^{\prime^{\prime}}}+\ldots\right)|\Theta\rangle(28)
\end{aligned}
$$

where $|\nu\rangle$ are the exact excited eigenstates at $\boldsymbol{h}_{s}(H|\nu\rangle=$ $\left.E_{\nu}|\nu\rangle,\langle\nu \mid \Theta\rangle=0\right)$, normally entangled, and $S_{i}^{-^{\prime}}=$ $R_{i} S_{i}^{-} R_{i}^{\dagger}$ the rotated lowering operators, with $\alpha, \beta_{i j}$ and all remaining terms of order $\delta h_{\perp}$ if $\boldsymbol{h}-\boldsymbol{h}_{s}=$ $\delta h_{\perp} \boldsymbol{n}_{\theta}^{\perp}+\delta h_{\|} \boldsymbol{n}_{\theta}$. In the rotated standard basis $\left\{\otimes_{i}\left|k^{\prime}{ }_{i}\right\rangle\right\}$ $\left(S_{z^{\prime}}^{i}\left|k^{\prime}{ }_{i}\right\rangle=\left(S_{i}-k\right)\left|k_{i}^{\prime}\right\rangle\right)$ and considering first $S=1 / 2$, 
Eq. (28) leads to

$$
\rho_{i j} \approx\left(\begin{array}{cccc}
1 & \alpha & \alpha & \beta_{i j} \\
\alpha & 0 & 0 & 0 \\
\alpha & 0 & 0 & 0 \\
\beta_{i j} & 0 & 0 & 0
\end{array}\right)+O\left(\delta h_{\perp}^{2}\right) .
$$

According to the positive partial transposition criterion 25, 26], this state will be entangled if its partial transpose $\rho_{i j}^{T_{j}}$ is non-positive, i.e., if it has at least one negative eigenvalue. But the the partial transpose of (29) has eigenvalues 1,0 and $\pm \beta_{i j}$ up to $O\left(\delta h_{\perp}\right)$, so that $\rho_{i j}$ will be entangled if $\beta_{i j} \neq 0$. And the exact coefficients $\beta_{i j}$ obtained from (28) are not strictly zero for any pair $i, j$ linked by successive applications of the couplings in $H$, due to the two spin excitations present in the exact eigenstates $|\nu\rangle$.

For higher spins $S, \rho_{i j}$ will be more complex (of $\left.(2 S+1)^{2} \times(2 S+1)^{2}\right)$ but will still contain a first submatrix of the form (29). Hence, it will also be entangled if $\beta_{i j} \neq 0$, since the partial transpose of this block is the first block of the full partial transpose $\rho_{i j}^{T_{j}}$, and is non-positive at $O\left(\delta h_{\perp}\right)$. This prevents the full $\rho_{i j}^{T_{j}}$ from being positive semidefinite (in which case all principal submatrices should also be so).

For $S=1 / 2$, the entanglement between spins $i$ and $j$ can be measured through the concurrence 27] $C_{i j}=$ $2 \lambda_{\max }-\operatorname{Tr} M_{i j}$, where $\lambda_{\max }$ is the largest eigenvalue of the matrix $M_{i j}=\left[\rho_{i j}^{1 / 2} \tilde{\rho}_{i j} \rho_{i j}^{1 / 2}\right]^{1 / 2}$, with $\tilde{\rho}_{i j}=\sigma_{y} \otimes$ $\sigma_{y} \rho_{i j}^{*} \sigma_{y} \otimes \sigma_{y}$ in the standard basis. Up to $O\left(\delta h_{\perp}\right)$, Eq. (29) then leads to

$$
C_{i j} \approx 2\left|\beta_{i j}\right| \propto\left|\delta h_{\perp}\right| .
$$

Note that at this order, $\alpha$ in (29) has no effect on the eigenvalues of $\rho_{i j}^{T_{j}}$ nor on $C_{i j}$. Eq. (30) implies that $C_{i j}$, while acquiring finite positive values in the neighborhood of $\boldsymbol{h}_{s}$, will vanish linearly (as $\left|\delta h_{\perp}\right|$ ) as $\boldsymbol{h} \rightarrow \boldsymbol{h}_{s}$, i.e., as it crosses the straight line of factorizing fields at a fixed direction $\boldsymbol{n}_{\gamma}$. The corresponding entanglement of formation [27], $E_{i j}=-\sum_{\nu= \pm} p_{\nu} \log _{2} p_{\nu}$, with $p_{ \pm}=\frac{1 \pm \sqrt{1-C_{i j}^{2}}}{2}$, is just a convex increasing function of $C_{i j}$, which vanishes as $-\frac{1}{4} C_{i j}^{2} \log _{2}\left(C_{i j}^{2} / 4 e\right)$ for $C_{i j} \rightarrow 0$. Hence, for $\boldsymbol{h} \rightarrow \boldsymbol{h}_{s}$ it will vanish essentially as $-\delta h_{\perp}^{2} \log _{2}\left|\delta h_{\perp}\right|$.

It is also seen from (29) that the eigenvalues of $\rho_{i j}$ will be either 1 (with negative $O\left(\delta h_{\perp}^{2}\right)$ corrections) or small

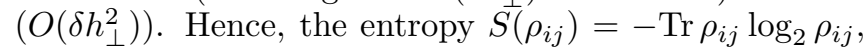
which measures the entanglement between the pair and the rest of the system 28], will also vanish essentially as $-\delta h_{\perp}^{2} \log _{2}\left|\delta h_{\perp}\right|$ for $\boldsymbol{h} \rightarrow \boldsymbol{h}_{s}$. The same behavior at $\boldsymbol{h}_{s}$ will be exhibited by the single spin entropy $S[\rho(1)]$, where $\rho(1)=\rho_{i}=\operatorname{Tr}_{j} \rho_{i j}$ denotes the single spin reduced state, and also by the block entropy 29] $S[\rho(m)]$ of $m$ contiguous spins, where $\rho(m)$ denotes their reduced state. Factorization can in fact be directly seen through the entanglement spectrum [19, 30, i.e. the set of eigenvalues of the reduced states $\rho(m)$. At $\boldsymbol{h}=\boldsymbol{h}_{s}, \rho(m)$ will have just one nonzero eigenvalue $p_{1}=1$, whereas in its vicinity the remaining eigenvalues will be small, of order $O\left(\delta h_{\perp}^{2}\right)$.

\section{B. Entanglement in the vicinity of the NGS}

We first recall that in the transverse case $\boldsymbol{h}=h \boldsymbol{n}_{z}$, the behavior of $C_{i j}$ close to the TFF $\boldsymbol{h}_{z s}$ in the GS of a finite FM-type chain [10, 11] is different from that described above. Since in the transverse case the $S_{z}$ parity $P_{z}$ is conserved, the exact GS of a finite spin chain has a definite parity, exhibiting parity transitions (the last one at the TFF $\boldsymbol{h}_{z s}$ ) as the transverse field is increased 10]. This implies that for $\boldsymbol{h} \rightarrow \boldsymbol{h}_{z s}^{ \pm}$, it actually approaches the entangled definite parity degenerate sidelimits $\left|\Theta^{ \pm}\right\rangle=\frac{|\Theta\rangle \pm|-\Theta\rangle}{\sqrt{2(1 \pm\langle-\Theta \mid \Theta\rangle)}}$, with $|-\Theta\rangle=P_{z}|\Theta\rangle$. These states lead to common finite side-limits $C_{ \pm}$of the concurrence $C_{i j}$ for any pair $i \neq j$, given for $S=1 / 2$ by 10]

$$
C^{ \pm}=\left|\frac{\sin ^{2} \theta \cos ^{n-2} \theta}{1 \pm \cos ^{n} \theta}\right|,
$$

where $n$ is the number of spins and $\cos \theta$ is determined by (13), with $\langle-\Theta \mid \Theta\rangle=\cos ^{n} \theta$. For finite $n$, a small but finite discontinuity in $C_{i j}$ is then encountered as the transverse field $\boldsymbol{h}$ crosses $\boldsymbol{h}_{z s}$, reflecting the parity change of the GS at $\boldsymbol{h}_{z s}$. Of course, exactly at $\boldsymbol{h}=\boldsymbol{h}_{z s}$, the GS is two-fold degenerate and entanglement depends on the choice of GS, as in general degenerate systems 31. Factorization implies that the minimum entanglement at this point is zero (obtained when choosing $| \pm \Theta\rangle$ as GS), even though the side-limits are finite.

Remarkably, in the AFM chain, Eq. (31) remains formally valid for the side-limits of $C_{i j}$ at the Néel NTFF $\boldsymbol{h}_{s}^{\prime}$, i.e., as $\boldsymbol{h}$ at a fixed orientation $\boldsymbol{n}_{\gamma}$ crosses the ellipsoid of factorizing fields $\boldsymbol{h}_{s}^{\prime}$. The reason is that the exact GS of a finite cyclic AFM chain in a uniform field preserves TI away from crossing points and hence, it approaches for $\boldsymbol{h} \rightarrow \boldsymbol{h}_{s}^{\prime \pm}$ the entangled TI side-limits

$$
\left|\Theta_{N}^{ \pm}\right\rangle=\frac{\left|\Theta_{N}\right\rangle \pm\left|-\Theta_{N}\right\rangle}{\sqrt{2\left(1 \pm\left\langle-\Theta_{N} \mid \Theta_{N}\right\rangle\right)}},
$$

where $\left|\Theta_{N}\right\rangle=\left|\theta_{1} \phi_{1}, \theta_{2} \phi_{2}, \ldots\right\rangle, \quad\left|-\Theta_{N}\right\rangle=$ $\left|\theta_{2} \phi_{2}, \theta_{1} \phi_{1}, \ldots\right\rangle=T\left|\Theta_{N}\right\rangle$ denote the degenerate Néel-type separable GS's at $\boldsymbol{h}_{s}^{\prime}$ ( $T$ denotes the one-site translation operator, with $\left.T\left|\Theta_{N}^{ \pm}\right\rangle= \pm\left|\Theta_{N}^{ \pm}\right\rangle\right)$. And these states $\left|\Theta_{N}^{ \pm}\right\rangle$lead to similar side-limits for the concurrence $C_{i j}$ between any two spins $i \neq j$ (see (35) below), i.e.,

$$
C^{ \pm}=\left|\frac{\sin ^{2} \theta^{\prime} \cos ^{n-2} \theta^{\prime}}{1 \pm \cos ^{n} \theta^{\prime}}\right|,
$$

where $\theta^{\prime}$ is half the difference between the alternating angles of the Néel solution. This angle is determined by 
[1]

$$
\cos ^{2} \theta^{\prime}=\frac{\left(J_{z}+J_{y}\right)\left(J_{x}+J_{y}\right)}{J_{x}+J_{z}} \frac{\left(J_{x}+J_{y}\right) \cos ^{2} \gamma+\left(J_{z}+J_{y}\right) \sin ^{2} \gamma}{\left(J_{x}+J_{y}\right)^{2} \cos ^{2} \gamma+\left(J_{z}+J_{y}\right)^{2} \sin ^{2} \gamma},
$$

if $|\gamma|<\gamma_{m}$ (Eq. (27)), as in the case of Fig. 4 ( $\cos ^{2} \theta^{\prime}$ is given by the inverse of (34) if $\gamma_{m}<\gamma<\pi-\gamma_{m}$ ). Hence, in finite chains small yet finite side-limits together with a discontinuity will be exhibited by the concurrences $C_{i j}$ as $\boldsymbol{h}$ crosses $\boldsymbol{h}_{s}^{\prime}$ at a fixed orientation, as verified in the top right panel of Fig. (4.

We can extend Eq. (33) to general spin $S>1 / 2$ by still considering the reduced states $\rho_{i j}^{ \pm}$arising from $\left|\Theta_{N}^{ \pm}\right\rangle$, as those of two effective qubits, stemming from the single site states $\left|\Theta_{N i}^{ \pm}\right\rangle,\left|\Theta_{N j}^{ \pm}\right\rangle$, as done in ref. 11] for the TFF. The generalized expression is obtained replacing $\cos \theta^{\prime} \rightarrow$ $\cos ^{2 S} \theta^{\prime}$ and $\sin ^{2} \theta^{\prime} \rightarrow 1-\cos ^{4 S} \theta^{\prime}$ in (33). The negativity can be similarly evaluated [1].

The side-limits at $\boldsymbol{h}_{s}^{\prime}$ of the reduced state of $m$ given spins, $\rho^{ \pm}(m)$, can be directly obtained from the exact side-limits (32) of the full GS. They will be rank 2 mixed states (and not rank one states, i.e., pure states, as in $\boldsymbol{h}_{s}$ ), of the form

$$
\begin{aligned}
& \rho^{ \pm}(m)= \\
& \frac{\left|\Theta_{N}^{m}\right\rangle\left\langle\Theta_{N}^{m}|+|-\Theta_{N}^{m}\right\rangle\left\langle-\Theta_{N}^{m}\right| \pm\left(\left|\Theta_{N}^{m}\right\rangle\left\langle-\Theta_{N}^{m}\right|\left\langle-\Theta_{N}^{n-m} \mid \Theta_{N}^{n-m}\right\rangle+h . c .\right)}{2\left(1 \pm\left\langle-\Theta_{N} \mid \Theta_{N}\right\rangle\right)},
\end{aligned}
$$

where $\left| \pm \Theta_{N}^{m}\right\rangle$ denote the reduced states of the $m$ spins in the Néel states $\left| \pm \Theta_{N}\right\rangle$ and $\left\langle-\Theta_{N} \mid \Theta_{N}\right\rangle=\cos ^{n} \theta^{\prime}$, $\left\langle-\Theta_{N}^{m} \mid \Theta_{N}^{m}\right\rangle=\cos ^{m} \theta^{\prime}$, with $\cos ^{2} \theta^{\prime}$ given by (34) for $|\gamma|<$ $\gamma_{m}$. The exact eigenvalues of $\rho^{ \pm}(m)$ are $p^{ \pm}(m)$ and $1-$ $p^{ \pm}(m)$, with

$$
p^{ \pm}(m)=\frac{\left(1+\cos ^{m} \theta^{\prime}\right)\left(1 \pm \cos ^{n-m} \theta^{\prime}\right)}{2\left(1 \pm \cos ^{n} \theta^{\prime}\right)} .
$$

The spectrum of $\rho(m)$ will then reduce to these two eigenvalues for $\boldsymbol{h} \rightarrow \boldsymbol{h}_{s}^{\prime \pm}$. These side-limits are independent of the choice of the $m$ spins, i.e., the same for $m$ contiguous or separated spins, as in the UGS of the transverse case 11]. For general spin $S$, we should just replace $\cos \theta^{\prime} \rightarrow \cos ^{2 S} \theta^{\prime}$ in (36).

The ensuing side-limits at $\boldsymbol{h}_{s}^{\prime}$ of the entanglement entropy $S[\rho(m)]$ are then

$S\left[\rho^{ \pm}(m)\right]=-p^{ \pm}(m) \log _{2} p^{ \pm}(m)-\left[1-p^{ \pm}(m)\right] \log _{2}\left[1-p^{ \pm}(m)\right]$.

For sufficiently large $m \leq n / 2$, the overlap $\left\langle-\Theta_{N}^{m} \mid \Theta_{N}^{m}\right\rangle$ vanishes and $p^{ \pm}(m) \rightarrow 1 / 2, S\left[\rho^{ \pm}(m)\right] \rightarrow 1$. For $m=2$ we also obtain from (35) the side-limits of the reduced state of a spin pair, which lead to the separation independent limits (33) of the concurrence.

\section{Discussion}

In Figs. 46 we show illustrative exact results for a cyclic FM (left) and AFM (right) spin 1/2 chain of $n=12$
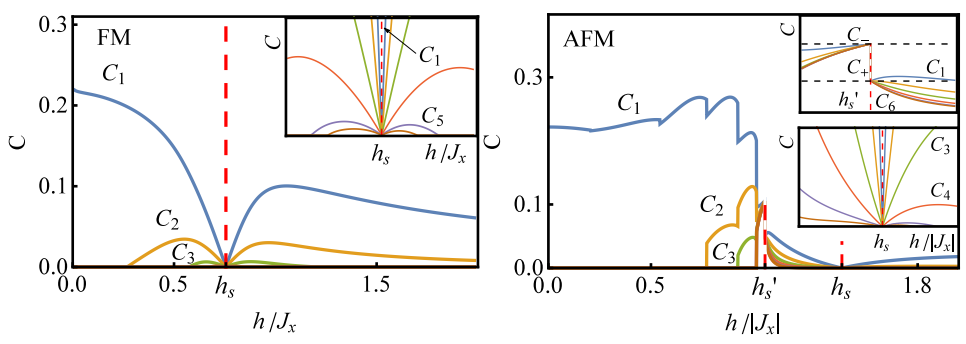

FIG. 4. (Color online) The concurrences $C_{l}$ between spins $i$ and $i+l$ (top) in a FM (left) and AFM (right) finite spin $1 / 2$ $X Y$ chain with $\chi=J_{y} / J_{x}=1 / 2$, as a function of the magnitude $h=|\boldsymbol{h}|$ of the non-transverse field at fixed orientation $\boldsymbol{n}_{\gamma}=(\sin \gamma, \cos \gamma)$ in the $x z$ plane, with $\gamma=0.02 \pi(\mathrm{FM})$ and $0.36 \pi(\mathrm{AFM})$. For these values there is a single factorizing field $\left|\boldsymbol{h}_{s}\right| \approx 0.76 J_{x}$ (Eq. (19) in the FM case, determining a UGS, and two factorizing fields $\left|\boldsymbol{h}_{s}^{\prime}\right| \approx 1.06\left|J_{x}\right|($ Eq. 26 ) and $\left|\boldsymbol{h}_{s}\right| \approx 1.43\left|J_{x}\right|$ in the AFM case, corresponding to a NGS and UGS respectively. The insets depict the details in the vicinity of these fields, showing that all $C_{l}$ 's vanish linearly at $\boldsymbol{h}_{s}$ (Eq. (30) and approach the finite $l$-independent side-limits (33) at $\boldsymbol{h}_{s}^{\prime}$. All pairs are entangled in the vicinity of $\boldsymbol{h}_{s}$ and $\boldsymbol{h}_{s}^{\prime}$, remaining so between both fields in the AFM case considered. All labels dimensionless.

spins interacting through first neighbor $X Y$ couplings $\left(J_{z}=0\right)$ with $\chi=J_{y} / J_{x}=1 / 2$, immersed in a nontransverse field, where all previous effects can be clearly appreciated and verified. The numerical results were obtained through diagonalization (notice that an exact analytic solution of the $X Y$ chain through the JordanWigner fermionization 32] is feasible just for transverse fields [14]). All quantities are depicted as a function of the scaled magnitude $|\boldsymbol{h}| /\left|J_{x}\right|$ of the non-transverse field at fixed orientation in the $x z$ plane $(\gamma=0.02 \pi$ in the FM case, $\gamma=0.36 \pi$ in the AFM case). For these orientations there is a single NTFF $\boldsymbol{h}_{s}$ in the FM case, determining a UGS, whereas in the AFM case there are two NTFF, the first one $\boldsymbol{h}_{s}^{\prime}$ corresponding to a NGS and the second one $\boldsymbol{h}_{s}$ to a UGS.

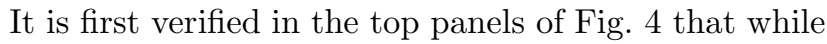
at weak fields just the first neighbor concurrence $C_{1}$ is finite in the present FM and AFM cases, all concurrences $C_{l}$ become non-zero in the proximity of the factorizing fields. As seen in the insets, in the vicinity of $\boldsymbol{h}_{s}$ their behavior is correctly described by Eq. (30), vanishing all linearly with $\left|\boldsymbol{h}-\boldsymbol{h}_{s}\right|$ for $\boldsymbol{h} \rightarrow \boldsymbol{h}_{s}\left(\beta_{i j} \propto \delta h_{\perp} \eta^{-|i-j|}(\eta>\right.$ 1 ) in the case of Fig. 团 changing sign as $\boldsymbol{h}$ crosses $\boldsymbol{h}_{s}$ ). On the other hand, in the AFM case they all approach the finite $l$-independent distinct side-limits (33) at $\boldsymbol{h}_{s}^{\prime}$ (here $\cos \theta^{\prime} \approx 0.92$ and $C^{-} \approx 0.11, C^{+} \approx 0.049$ ). Both factorizing fields appear successively as the field increases along orientations $\theta<\gamma<\gamma_{m}$, leading to a rather broad interval of "long-range" pairwise entanglement located between $\boldsymbol{h}_{s}^{\prime}$ and $\boldsymbol{h}_{s}$, as appreciated in the right panel. It is also seen that all $C_{l}$ exhibit jumps for $|\boldsymbol{h}|<\left|\boldsymbol{h}_{s}^{\prime}\right|$, the last one at $\boldsymbol{h}_{s}^{\prime}$, which reflect the $n / 2$ translational parity transitions of the exact GS, as discussed below. We remark that while the side-limits (33) at $\boldsymbol{h}_{s}^{\prime}$ diminish 

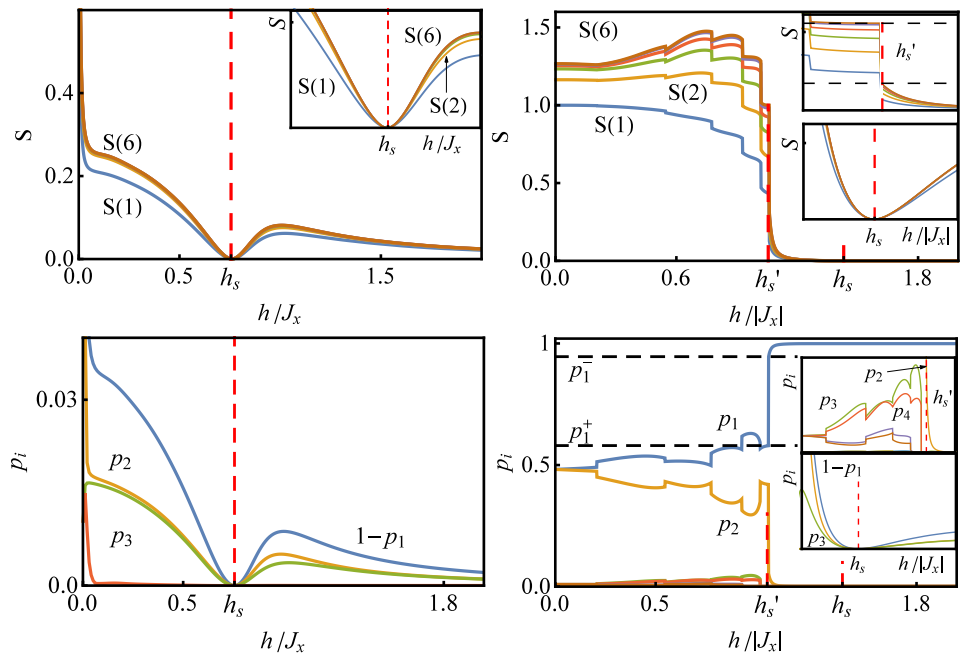

FIG. 5. The block entropies $S(m)=S[\rho(m)]$ of $m$ contiguous spins (top) and the eigenvalues $p_{i}$ (entanglement spectrum) of the corresponding reduced states $\rho(m)$ for $m=5$, in the same FM (left) and AFM (right) systems of Fig. 4 At $\boldsymbol{h}_{s}$ (UGS), $S(m)$ and all but one $\left(p_{1}\right)$ of the eigenvalues of $\rho(m)$ vanish, while for $\boldsymbol{h} \rightarrow \boldsymbol{h}_{s}^{\prime \pm}$ (NGS), $S(m)$ approaches the finite sidelimits (37) (indicated for $m=n / 2)$, and two eigenvalues $\left(p_{1}\right.$ and $p_{2}$ ) remain nonzero, with $p_{1}$ approaching the indicated side-limits (36). The insets depict again the details in the vicinity of the factorizing fields.

as the number $n$ of spins increases, the finite values of the $C_{l}$ 's in the vicinity of both $\boldsymbol{h}_{s}^{\prime}$ and $\boldsymbol{h}_{s}$ persist for larger sizes.

The block entanglement entropies of $m$ contiguous spins are depicted in Fig. 5. Block entropies in $X Y$ or $X Y Z$ spin chains have been studied in detail just for zero or transverse fields 19, 29, 33], including also block Renyi entropies 19, 34, 35]. It is first verified that the von Neumann entropies $S[\rho(m)]$ vanish essentially quadratically in the vicinity of $\boldsymbol{h}_{s}$, whereas in the AFM case they approach the finite side-limits (37) at the Néel factorizing field $\boldsymbol{h}_{s}^{\prime}$ (here $S\left[\rho^{+}(m)\right] \approx 0.31$ while $S\left[\rho^{-}(m)\right]=1$ for $m=n / 2$; notice from (36) that $p^{-}(m)=1 / 2$ for $m=n / 2 \forall n)$. In the FM case these entropies rapidly saturate as $m$ increases for all non-zero fields, showing then a non-critical behavior, whereas in the AFM case, while above $\left|\boldsymbol{h}_{s}\right|$ they become small $(<0.01)$ and also rapidly saturate, below $\left|\boldsymbol{h}_{s}^{\prime}\right|$ they are larger and show an appreciable dependence with block size.

The behavior of these entropies can be better understood by means of the entanglement spectrum, shown in the bottom panels, where the eigenvalues $p_{i}$ of $\rho(m)$ for $m=5$ are depicted. Results for other $m>1$ are similar. In the FM case, there are three dominant eigenvalues, with $p_{1}$ close to 1 , and the behavior of $p_{2}$ and $p_{3}$ resembles that of $S(m)$ : All eigenvalues except $p_{1}$ vanish (quadratically) at $\boldsymbol{h}_{s}$. However, in the AFM case it is seen that for $|\boldsymbol{h}|<\left|\boldsymbol{h}_{s}^{\prime}\right|$, both $p_{1}$ and $p_{2}$ are significant and comparable, indicating roughly an approximate rank 2 reduced state. When $\boldsymbol{h} \rightarrow \boldsymbol{h}_{s}^{\prime \pm}, \rho(m)$ becomes exactly
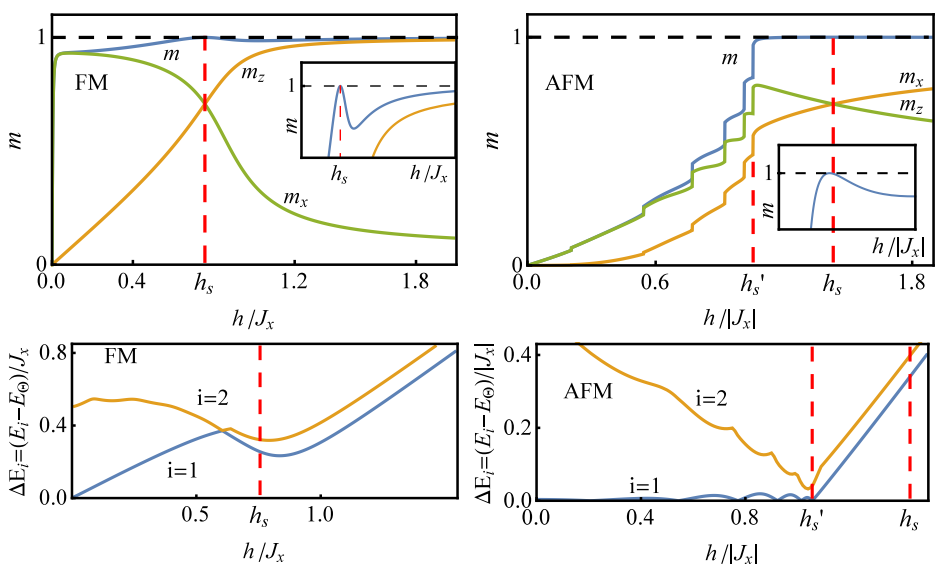

FIG. 6. (Color online) The scaled intensive magnetizations $m_{\mu}=\sum_{i} 2\left\langle S_{i}^{\mu}\right\rangle / n$ and $m \equiv|\boldsymbol{m}|$ (top) and the scaled energy gap between the ground and the first two excited states (bottom) in the same FM (left) and AFM (right) systems of Fig. 4. Notice that $m=1$ at the NTFF $\boldsymbol{h}_{s}$ determining the UGS, as seen in the insets. The energy gap shows that the UGS is well separated from the first excited state, while the NGS is two-fold degenerate. All labels dimensionless.

a rank 2 state and just $p_{1}$ and $p_{2}$ are nonzero, in agreement with Eqs. (36) - (35). The behavior is similar to that observed for a transverse field [19]. As expected, the GS transitions taking place in this sector are clearly visible in both the block entropy and the entanglement spectrum. For $|\boldsymbol{h}|>\boldsymbol{h}_{s}^{\prime}$ there are just three dominant eigenvalues, with $p_{1}$ much larger than the rest, as in the FM case. All but $p_{1}$ vanish again at the second factorizing field $\boldsymbol{h}_{s}$.

Let us remark that at zero field, the results for any entanglement measure in the FM and AFM $X Y$ chains with first neighbor couplings are strictly coincident, since the corresponding Hamiltonians can be transformed into each other by a local rotation of angle $\pi$ around the $z$ axis at all even sites, which does not affect entanglement measures. This fact explains the pronounced increase of the block entropies of the FM case as the field vanish, since they approach in this limit the higher AFM values. This symmetry no longer holds for finite fields not pointing along the $z$ axis.

In spin $1 / 2$ systems, the magnetization can be used as a separability witness: The quantity $m=2 \sum_{i}\left|\left\langle\boldsymbol{S}_{i}\right\rangle\right| / n$ satisfies $m<1$ in any pure entangled state of such system, with $m=1$ if and only if the pure state is completely separable. For a state with TI, $m=2|\boldsymbol{M}| / n$, with $\boldsymbol{M}=\left\langle\sum_{i} \boldsymbol{S}_{i}\right\rangle$ the total magnetization. Hence $m=1$ at the NTFF $\boldsymbol{h}_{s}$, as verified in the top panels of Fig. 5 . entailing a non-monotonous behavior of $m$ for increasing fields, as seen in the insets. We have numerically checked that such non-monotonous behavior persists for larger sizes, indicating that it is not a finite size effect. Therefore, through a careful measurement of $\boldsymbol{M}$ or the associated susceptibility as a function of the applied field, one could be able to identify the NTFF $\boldsymbol{h}_{s}$.

In the bottom panels of Fig. $[$ it is seen that the UGS 


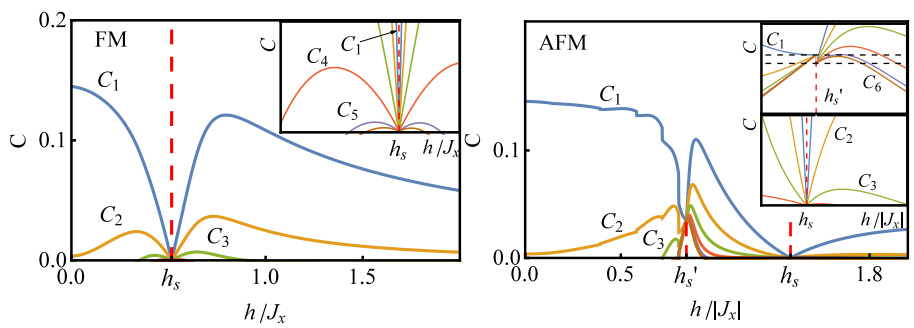

FIG. 7. (Color online) The concurrences $C_{l}$ between spins $i$ and $i+l$ in FM and AFM $X Y Z$ chains with $J_{y} / J_{x}=1 / 2$, $J_{z}=0.2\left|J_{x}\right|$ and $J_{x}>0(<0)$ in the left (right) panel. The orientation of the applied magnetic field in each panel is the same as that of Figs. 44 The factorizing fields are now $\left|\boldsymbol{h}_{s}\right| \approx$ $0.52 J_{x}$ in the FM case, where $\chi=0.375$, and $\left|\boldsymbol{h}_{s}\right| \approx 1.39 J_{x}$, $\left|\boldsymbol{h}_{s}^{\prime}\right| \approx 0.84 J_{x}$ in the AFM case, where $\chi=0.583$. The sidelimits (33) at $\boldsymbol{h}_{s}^{\prime}$ and the linear vanishing of all concurrences at $\boldsymbol{h}_{s}$ are again verified.

at $\boldsymbol{h}_{s}$ is non-degenerate and well separated from the first excited state, whereas the NGS at $\boldsymbol{h}_{s}^{\prime}$ is two-fold degenerate. Actually, as seen from the energy gap and also from the magnetization and previous entanglement measures, while no transitions are observed in the FM case, in the AFM case the exact GS exhibits $n / 2$ transitions as $|\boldsymbol{h}|$ increases at fixed $\gamma$, the last one taking place at $\boldsymbol{h}_{s}^{\prime}$. They correspond to "translational parity" transitions $\left|G S^{ \pm}\right\rangle \rightarrow\left|G S^{\mp}\right\rangle$, with $\left|G S^{ \pm}\right\rangle$the exact TI ground states, which satisfy $T\left|G S^{ \pm}\right\rangle= \pm\left|G S^{ \pm}\right\rangle$( $T$ is the onesite translation operator). These transitions are similar to those observed for transverse fields in both AFM or FM systems 10, 11, 14, 19], where they are related with spin parity transitions and also end at the corresponding transverse factorizing field [10, 11, 14. Hence, $\boldsymbol{h}_{s}^{\prime}$ still represents, in the non-transverse case, a critical field for the finite system, indicating the passage to a different regime.

Finally, we depict in Fig. 7 results for the pairwise concurrence in a chain with full $X Y Z$ couplings, a system which cannot be mapped to independent fermions even in the transverse case $(32,35])$. We have set $J_{z}=0.2\left|J_{x}\right|$, in both the FM $\left(J_{x}>J_{y}>0\right)$ and $\operatorname{AFM}\left(J_{x}<J_{y}<0\right)$ cases, using the same previous field orientations. The behavior is quite similar to that of Fig. [4 with the GS translational parity transitions also present in the AFM case. One just notes the higher values of $C_{l}$ above the factorizing fields in both cases, and the closer side-limits at $\boldsymbol{h}_{s}^{\prime}$ in the AFM case (now $C^{-} \approx 0.036, C^{+} \approx 0.032$ ), due to the different value of the anisotropy ratio $\chi$. The values at zero field are again still strictly coincident due to the same sign of $J_{z}$. Results for the block entropy and entanglement spectrum for the finite case considered are also qualitatively similar to the previous results.

\section{CONCLUSIONS}

We have first determined the general conditions for the existence of separable eigenstates with maximum spin at each site in general arrays with anisotropic $X Y Z$ couplings immersed in a non-transverse field. The set of factorizing fields can be characterized by the local fields orthogonal to the local alignment direction, plus arbitrary fields parallel to the latter. We have next identified the possibility of a uniform non-degenerate separable GS in quite general systems of arbitrary spin, including FM and AFM-type chains and arrays, for fields parallel to a principal plane (Fig. 2). The coupling range can be arbitrary, provided the anisotropy ratio $\chi$ is constant. In AFM $X Y Z$ chains with first neighbor couplings, this separable solution coexists in field space with the Néel-type separable solution.

We have also demonstrated, for arbitrary spin, that pairwise entanglement reaches full range in a finite array in the vicinity of the factorizing field determining the uniform solution, with the concurrence vanishing linearly at this field. Full range is also reached at the Néel NTFF, although here it was shown that in finite cyclic even chains, the pairwise concurrence reaches finite side-limits in its vicinity, which were analytically evaluated. This NTFF was shown to correspond to the last parity transition of the GS in the finite cyclic chain. Block entropies were also analyzed and shown to vanish quadratically at the uniform NTFF, while reaching again finite (and analytically determined) side-limits at the Néel NTFF in these finite chains.

Present results and limits are also applicable to more complex systems, like dimerized chains and arrays 12, 14, 36. The recent possibility of performing quantum simulations of spin chains and lattices with tunable couplings through cold atoms in optical lattices 37 39] or trapped ions 39 43] augments the potential of the present results. Such experiments could then provide valuable insights into the remarkable phenomenon of factorization and its relation with entanglement and criticality in finite many body systems.

\section{ACKNOWLEDGMENTS}

The authors acknowledge support of CONICET (MC, NC) and CIC (RR) of Argentina.
[1] J. Kurmann, H. Thomas, and G. Müller, Physica A 112, 235 (1982).

[2] G. Müller and R.E. Shrock, Phys. Rev. B 32, 5845 (1985).
[3] T. Roscilde et al., Phys. Rev. Lett. 93, 167203 (2004).

[4] T. Roscilde et al., Phys. Rev. Lett. 94, 147208 (2005).

[5] L. Amico et al., Phys. Rev. A 74, 022322 (2006).

[6] F. Baroni, A. Fubini, V. Tognetti, P. Verrucchi, J. Phys. 
A 40, 9845 (2007).

[7] S.M. Giampaolo, F. Illuminati, P. Verrucchi, S. De Siena, Phys. Rev. A 77, 012319 (2008).

[8] S.M. Giampaolo, G. Adesso, and F. Illuminati, Phys. Rev. Lett. 100, 197201 (2008).

[9] S.M. Giampaolo, G. Adesso, and F. Illuminati, Phys. Rev. B 79, 224434 (2009).

[10] R. Rossignoli, N. Canosa, and J.M. Matera, Phys. Rev. A 77, 052322 (2008).

[11] R. Rossignoli, N. Canosa, and J.M. Matera, Phys. Rev. A 80, 062325 (2009).

[12] G.L. Giorgi, Phys. Rev. B 79, 060405(R) (2009).

[13] S.M. Giampaolo, G. Adesso, and F. Illuminati, Phys. Rev. Lett. 104, 207202 (2010).

[14] N. Canosa, R. Rossignoli, and J.M. Matera, Phys. Rev. B 81, 054415 (2010).

[15] L. Ciliberti, R. Rossignoli, and N. Canosa, Phys. Rev. A 82, 042316 (2010).

[16] M. Rezai, A. Langari, J. Abouie, Phys. Rev. B 81, 060401R (2010); J. Abouie, M. Rezai, A. Langari, Prog. Theor. Phys. 127315 (2012).

[17] B. Tomasello, D. Rossini, A. Hamma, L. Amico, Europhys. Lett. 96, 27002 (2011).

[18] S. Campbell, J. Richens, N.L. Gullo, T. Busch, Phys. Rev. A 88, 062305 (2013).

[19] S.M. Giampaolo, S. Montangero, F. Dell' Anno, S. De Siena, and F. Illuminati, Phys. Rev. B 88, 125142 (2013).

[20] G. Karpat, B. Cakmak, and F.F. Fanchini, Phys. Rev. B 90, 104431 (2014).

[21] M.A. Nielsen and I. Chuang, Quantum Computation and Quantum Information, Cambridge Univ. Press, UK (2000).

[22] V. Coffman, J. Kundu, and W.K. Wootters, Phys. Rev. A 61, 052306 (2000).

[23] T.J. Osborne and F. Verstraete, Phys. Rev. Lett. 96, 220503 (2006).

[24] R.F. Werner, Phys. Rev. A 40, 4277 (1989).
[25] A. Peres, Phys. Rev. Lett. 77, 1413 (1996).

[26] M. Horodecki, P. Horodecki, and R. Horodecki, Phys. Lett. A 223, 1 (1996).

[27] S. Hill and W.K. Wootters, Phys. Rev. Lett. 78, 5022 (1997); W.K. Wootters, Phys. Rev. Lett. 80, 2245 (1998).

[28] C.H. Bennett, H.J. Bernstein, S. Popescu, B. Schumacher, Phys. Rev. A 53, 2046 (1996).

[29] G. Vidal, J.I. Latorre, E. Rico, A. Kitaev, Phys. Rev. Lett. 90, 227902 (2003).

[30] H. Li and F.D.M. Haldane, Phys. Rev. Lett. 101, 010504 (2008).

[31] O.A. Castro-Alvaredo and B. Doyon, Phys. Rev. Lett. 108, 120401 (2012).

[32] E. Lieb, T. Schultz, and D. Mattis, Ann. Phys. (N.Y.) 16, 407 (1961).

[33] A.R. Its, B-Q. Jin, V.E. Korepin, J. Phys. A 38, 2975 (2005); F. Franchini, A.R. Its, B-Q. Jin, V.E. Korepin, J. Phys. A 40, 8467 (2007).

[34] F. Franchini, A.R. Its, V.E. Korepin, J. Phys. A 41, 025302 (2008).

[35] E. Ercolessi, S. Evangelisti, F. Franchini, F. Ravanini, Phys. Rev. B 83, 012402 (2011).

[36] A. Boette, R. Rossignoli, N. Canosa, and J.M. Matera, Phys. Rev. B 91, 064428 (2015).

[37] J. Simon et al, Nature 474, 307 (2011).

[38] M. Lewenstein, A. Sanpera, and V. Ahufinger, Ultracold Atoms in Optical Lattices, Oxford Univ. Press, UK (2012).

[39] I. M. Georgescu, S. Ashhab, and F. Nori, Rev. Mod. Phys. 86, 153 (2014).

[40] D. Porras and J.I. Cirac, Phys. Rev. Lett. 92, 207901 (2004).

[41] K. Kim et al, Phys. Rev. Lett. 103, 120502 (2009).

[42] R. Blatt and C.F. Roos, Nature Physics 8, 277 (2012).

[43] S. Korenblit et al, New J. Phys. 14, 095024 (2012). 